\title{
Effects of ruminant digestion and metabolism on phenolic monomers of forages
}

\author{
BY HANS-JOACHIM G. JUNG*, GEORGE C. FAHEY JR† \\ AND NEAL R. MERCHEN \\ Department of Animal Science, University of Illinois, Urbana, IL 61801, USA
}

\section{(Received 20 September 1982 - Accepted 6 June 1983)}

1. Immature and mature lucerne (Medicago sativa) and tall fescue (Festuca arundinacea) hays were analysed for their lignin and phenolic monomer (hydroxybenzoic and hydroxycinnamic acid derivatives) contents. These hays were given to four sheep with rumen, duodenal and ileal cannulas, and to a steer with rumen and abomasal cannulas, to investigate the extent and sites of digestion of lignin and phenolic monomers.

2. The hays and digesta samples were analysed for alkali and nitrobenzene-extractable phenolic monomers. Lanthanum oxide was used as an external marker in the digestion studies. Urine was also collected for estimates of total phenolic balance.

3. Grass hays contained greater concentrations of alkali-labile phenolic monomers than did legume hays, whereas legume hays had higher levels of nitrobenzene oxidation products. Mature tall fescue hay had greater concentrations of phenolic monomers than did immature tall fescue hay, but corresponding differences with maturity were not observed with lucerne hays.

4. There were quantitative differences between diets in the digestibilities and in the sites of digestion of phenolic monomers. Digestibilities ranged from 0.191 to 1.004 for alkali-labile compounds and from -1.137 to 0.868 for nitrobenzene oxidation products. Composition of lignin was altered during its passage through the digestive tract. The proportion of phenolic monomers not recovered in urine and faeces varied from -1.014 to 0.871 for individual compounds and differed between diets. Soluble phenolic monomers decreased in concentration as digesta passed from the rumen to the abomasum and duodenum, but increased again in the ileum; levels varied with diet. Phenolics were rapidly solubilized in the rumen after feeding.

5. Results indicate that forages differ in their phenolic monomer content and in the digestibility of these compounds. Therefore, lignin is not an inert compound in the digestive tract of ruminants as has been previously reported.

Although lignification is generally considered to be the primary factor limiting the digestion of forage cell-wall carbohydrates by ruminants, relatively little attention has been given to the differences in lignin composition of forages and how those differences might account for variations between plant species in lignin's inhibitory effects. Recent work (Akin, 1982; Jung \& Fahey, 1983b) demonstrated that the phenolic monomer constituents of lignin are inhibitors of cellulose degradation by rumen microbes in vitro and that phenolic compounds differ in their extent of inhibition. Forages are known to differ in their content of these phenolic monomers (Gee et al. 1968; Hartley \& Jones, 1977; Jung \& Fahey, 1983a). Degradation of forage cell walls by rumen micro-organisms results in alterations in the amounts of phenolics recoverable from the plant material, with apparent digestibilities being noted for most monomers (Theander et al. 1981; Jung et al. 1983).

Intake of feedstuffs by non-ruminants is depressed by the presence of phenolic monomers (hydroxybenzoic and hydroxycinnamic acid derivatives) in the diet (Joslyn \& Glick, 1969; Jung \& Fahey, 1983c). Absorption of phenolics, and presumably their effect on metabolism, accounts for at least a portion of this intake reduction (Glick, 1981). Ruminants also absorb phenolic compounds from their diets and excrete various phenolics in the urine (Martin, 1982).

\footnotetext{
- Present address: Roman L. Hruska U.S. Meat Animal Research Center, Clay Center, NE 68933, USA.

$\dagger$ For reprints.
} 
The purpose of the study reported in the present paper was to examine the changes which phenolic monomers of forages undergo during digestion. Cannulated animals were used to allow the apparent digestion of phenolics to be partitioned between the various sections of the gastrointestinal tract. Changes in the phenolic composition of the diet were related to potential effects on microbial activity in the rumen and animal performance.

\section{MATERIALS AND METHODS}

\section{Experimental design}

Expt 1. This experiment was designed to determine the apparent digestibility of phenolic monomers (derivatives of hydroxycinnamic and hydroxybenzoic acids) of forages in the various sections of the ruminant digestive tract. Four Suffolk wether sheep with rumen, duodenal and ileal cannulas were used in a $4 \times 4$ Latin-square metabolism trial. Duodenal cannulas were located approximately $50 \mathrm{~mm}$ posterior to the pyloric sphincter and the ileal cannulas were placed a similar distance anterior to the ileocaecal junction. Both intestinal cannulas were ' $T$ ' type. Animals were housed in metabolism cages in a controlledenvironment room. Each period consisted of a $14 \mathrm{~d}$ adaptation phase followed by a $7 \mathrm{~d}$ collection of urine and faeces. Animals were fed twice daily (08.00 and 20.00 hours). The diets were immature lucerne (Medicago sativa) hay (bud stage), mature lucerne hay (full bloom), immature tall fescue (Festuca arundinacea) hay (leafy regrowth) and mature tall fescue hay (full bloom). All diets contained $143 \mathrm{~g}$ glucose $/ \mathrm{kg}$ which was added as a $200 \mathrm{~g} / \mathrm{l}$ solution to the diets at feeding to increase the palatability of the tall fescue hays. The tall fescue diets also contained sufficient soya-bean meal to provide $120 \mathrm{~g}$ crude protein (nitrogen $\times 6.25) / \mathrm{kg}$ dry matter $(\mathrm{DM})$. All hays were ground to pass through a $9.5 \mathrm{~mm}$ screen. Hays were marked with approximately $100 \mathrm{mg}$ lanthanum oxide $/ \mathrm{kg}$ diet as described by Hartnell \& Satter (1979). Intakes were equal and limited such that no feed refusal occurred. Intakes were $700,817,1050$ and $1050 \mathrm{~g} \mathrm{DM} / \mathrm{d}$ in experimental periods $1,2,3$ and 4, respectively. Digesta samples were collected from the rumen, duodenum and ileum over a $2 \mathrm{~d}$ period such that a sample was obtained for each even hour of the day. Rumen samples were taken with a core sampler from various sites in the rumen, with six to eight cores being required to collect a $200 \mathrm{ml}$ sample. Duodenal digesta $(100-150 \mathrm{ml}$ per sample) and ileal digesta $(50-100 \mathrm{ml}$ per sample) were collected. Samples were frozen immediately.

Expt 2. A second experiment was conducted to monitor changes in the concentrations of soluble phenolic monomers in the rumen and abomasum with time after feeding. An Angus $\times$ Hereford steer with rumen and abomasal cannulas was given $6 \mathrm{~kg}$ of the immature lucerne once daily. After a $14 \mathrm{~d}$ adaptation period, rumen and abomasal digesta samples were taken at various times post-feeding. The steer was allowed to consume the diet for $1 \mathrm{~h}$ and feed remaining was then removed. Samples of digesta were taken at 0, 4, 8, 12 and $24 \mathrm{~h}$ post-feeding. Time zero was immediately after feed removal. Rumen samples were taken with a core sampler at various sites in the rumen. Rumen contents (1 1) and abomasal contents $(300 \mathrm{ml})$ were collected at each sampling time. Samples were frozen immediately, This sampling scheme was repeated three times with intervals of $1 \mathrm{~d}$ between samplings.

\section{Sample analysis}

Digesta samples from the sheep were composited to give samples representative of each collection site. Because of unequal sample volumes for the intestinal samples, $25 \mathrm{ml}$ digesta from each sample were used to make the composite sample. All digesta samples were centrifuged at $6000 \mathrm{~g}$ for $60 \mathrm{~min}$. The resulting pellet was taken to represent digesta solids in Expt 1. Rumen solids were not analysed as a representative sample of rumen solids could not be obtained. The supernatant fraction from centrifugation was filtered and the filtrate 
was taken to represent the liquid phase of digesta. Only liquid phase samples were retained from Expt 2. Duplicate $25 \mathrm{ml}$ portions of the liquid phase of digesta and of urine samples from Expt 1 were lyophilized, as were $50 \mathrm{ml}$ liquid phase samples from Expt 2. Digesta solids and faeces were also lyophilized. Diet samples, dried solids and faeces were ground to pass a $1 \mathrm{~mm}$ screen.

Diets and digesta solids were analysed for cell-wall constituents, lignin and phenolic monomers. Cell-wall constituents were analysed as neutral-detergent fibre, and lignin by extraction of samples with detergent followed by sulphuric acid $(720 \mathrm{ml} / \mathrm{l}$ ) (Goering \& Van Soest, 1970). Diet and digesta solids samples were analysed for both alkali-labile phenolic monomers and nitrobenzene oxidation products. The phenolic monomers identified in alkaline extracts of the samples exist as esters of both cell-wall carbohydrates and simple sugars of the cell-soluble fraction of plants (Jung et al. 1983). However, the majority of these phenolics are attached to the cell wall. Nitrobenzene oxidation is a standard analytical technique for lignin and, therefore, phenolic monomers released by this procedure are presumably derived from lignin. Samples $(500 \mathrm{mg}$ ) were extracted with $20 \mathrm{ml}$ anaerobic $1 \mathrm{M}$-sodium hydroxide for $24 \mathrm{~h}$ on a shaker bath in the absence of U.V. light. Sample preparation followed Hartley (1971) with samples being dissolved in $10 \mathrm{ml}$ methanol as the final solvent. Lyophilized samples of digesta liquid phase and urine were extracted with $20 \mathrm{ml}$ diethyl ether for $24 \mathrm{~h}$ on a shaker. Extracts were removed by filtration and the diethyl ether evaporated. The residues from the diethyl ether were redissolved in $1 \mathrm{ml}$ methanol. The diethyl ether-insoluble residues from digesta and urine were extracted under anaerobic conditions with $20 \mathrm{ml} 1 \mathrm{M}-\mathrm{NaOH}$ as described previously, the final solvent being $5 \mathrm{ml}$ methanol. The methanol extracts from samples were analysed for ten phenolic monomers ( $p$-coumaric, ferulic, gallic, $p$-hydroxybenzoic, protocatechuic, syringic and vanillic acids, $p$-hydroxybenzaldehyde, syringaldehyde, vanillin). A modification of the high-pressure liquid chromatographic technique of Hartley \& Buchan (1979) was used. Samples (40 $\mu$ l) were injected into a Hewlett-Packard 1084B liquid chromatograph (Hewlett-Packard, Palo Alto, CA) with a $250 \times 4.6 \mathrm{~mm}$ reverse phase column packed with Spherisorb- $\mathrm{C}_{18}(5 \mu \mathrm{m}$; Supelco, Inc., Bellefonte, PA). The solvent consisted of water-glacial acetic acid-butanol (350:1:7, by vol.) which was pumped isocratically at $3 \mathrm{ml} / \mathrm{min}$. The column temperature was $30^{\circ}$. The variable wavelength detector was programmed at $276 \mathrm{~nm}$ for the first $6 \mathrm{~min}$ of each determination and $300 \mathrm{~nm}$ for the remainder of the determination. Total analysis time for a determination was $17 \mathrm{~min}$. Identification and quantification of phenolic monomers was based on retention times and calibration of standard solutions of the phenolics.

Diet, digesta solids and faecal samples were analysed for lanthanum content by neutron activation (Gray \& Vogt, 1974). Digestibilities of DM, cell walls, lignin, alkali-labile phenolic monomers and nitrobenzene oxidation products were calculated using the marker ratio method.

\section{Statistical analysis}

Results from Expt 1 were analysed as a $4 \times 4$ Latin-square design. Expt 2 was analysed as a completely randomized design with three replicates. Means were compared by the least significant difference method for those factors having a significant analysis of variance.

\section{RESULTS}

\section{Composition of grass and lucerne hays}

As glucose will be rapidly fermented in the rumen, the DM associated with the glucose solution used to stimulate intake has been ignored in calculations of feed composition and diet digestibility. Table 1 presents the compositions of the diets used in Expt 1. Cell-wall content of the immature lucerne and tall fescue hays was less $(P<0.05)$ than that of mature 
Table 1. Expt 1. Composition $(\mathrm{g} / \mathrm{kg}$ ) of lucerne (Medicago sativa) and tall fescue (Festuca arundinacea) diets given to sheep

\begin{tabular}{llcccc}
\hline \hline \multicolumn{1}{c}{ Diet } & Soya-bean meal & Crude protein* & Cell wall & Lignin \\
\hline \multirow{2}{*}{ Lucerne hay: } & Immature & 0 & 201 & $530^{a}$ & $82^{a}$ \\
\multirow{2}{*}{ Tall fescue hay $:$} & Mature & 0 & 158 & $618^{b}$ & $114^{b}$ \\
\multirow{2}{*}{ SEM } & Mature & 124 & 120 & $587^{c}$ & $39^{c}$ \\
& & & 120 & $624^{b}$ & $42^{c}$ \\
\hline \hline
\end{tabular}

$a, b, c$ Mean values in the same vertical column not sharing common superscript letters differed significantly $(P<0.05)$.

* Nitrogen $\times 6.25$.

Table 2. Expt 1. Concentrations of alkali-labile phenolic monomers in the diets

\begin{tabular}{|c|c|c|c|c|c|c|c|c|}
\hline \multirow[b]{2}{*}{ Diet } & & \multicolumn{7}{|c|}{ Compound (mg/kg DM) } \\
\hline & & PCA & FA & PHBA & PHBAL & PA & VA & VAN \\
\hline \multirow[t]{2}{*}{ Lucerne hay: } & Immature & $570 \cdot 7^{a}$ & $1039 \cdot 7^{a}$ & $38 \cdot 7$ & $13 \cdot 9^{a}$ & $39 \cdot 0^{a}$ & $9 \cdot 7^{a}$ & $23-0^{a}$ \\
\hline & Mature & $628 \cdot 1^{a}$ & $978 \cdot 6^{\alpha}$ & $56 \cdot 5$ & $21 \cdot 0^{a}$ & $30 \cdot 9^{a}$ & $16 \cdot 8^{a}$ & $69 \cdot 3^{a b}$ \\
\hline \multirow[t]{2}{*}{ Tall fescue hay: } & Immature & $1834 \cdot 1^{b}$ & $1786 \cdot 4^{b}$ & $50 \cdot 0$ & $46 \cdot 5^{b}$ & $71 \cdot 4^{b}$ & $40 \cdot 4^{b}$ & $86-7^{b c}$ \\
\hline & Mature & $3025 \cdot 7^{c}$ & $2394 \cdot 8^{c}$ & $39 \cdot 5$ & $58 \cdot 9^{c}$ & $34 \cdot 4^{a}$ & $53 \cdot 6^{b}$ & $130 \cdot 2^{c}$ \\
\hline SEM & & $38 \cdot 1$ & $65 \cdot 6$ & 5.9 & $3 \cdot 2$ & 5.9 & 3.4 & $16 \cdot 1$ \\
\hline
\end{tabular}

PCA, $p$-coumaric acid; FA, ferulic acid; PHBA, $p$-hydroxybenzoic acid; PHBAL, $p$-hydroxybenzaldehyde; PA, protocatechuic acid; VA, vanillic acid; VAN, vanillin; DM, dry matter.

$a, b, c$ Mean values in the same vertical column not sharing common superscript letters differed significantly $(P<0.05)$.

harvests of these forages. Lignin content of mature lucerne hay was greater $(P<0.05)$ than that of immature lucerne, whereas the two tall fescue hays did not differ (Table 1). Grass hays generally had higher concentrations of alkali-labile phenolic monomers than did legume hays (Table 2). Only $p$-hydroxybenzoic acid levels did not differ between diets. Immature and mature lucerne hays did not differ significantly in their content of phenolic monomers. In contrast, mature tall fescue hay had higher $(P<0.05)$ levels of $p$-coumaric acid, ferulic acid and $p$-hydroxybenzaldehyde than did immature tall fescue hay. However, the concentration of protocatechuic acid was greater $(P<0.05)$ for immature than for mature tall fescue (Table 2). Alkali-labile phenolic monomers are presented as $\mathrm{mg} / \mathrm{kg} \mathrm{DM}$ (Table 2) because it has been shown that a small, but significant, proportion of these compounds are associated with the cell-soluble fraction of plants (Jung et al. 1983). Nitrobenzene oxidation products of the alkali-insoluble plant material generally represent only phenolic monomers of lignin. Their concentrations in diets are presented on a $\mathrm{g} / \mathrm{kg}$ lignin basis in Table 6 (p. 644). Composition of the two lucerne lignins did not differ significantly, whereas mature tall fescue lignin had greater $(P<0.05)$ levels of $p$-coumaric acid, $p$-hydroxybenzaldehyde, syringic acid, syringaldehyde, vanillic acid and vanillin than did lignin of immature tall fescue hay. $p$-Hydroxybenzoic acid levels were higher $(P<0.05)$ in the immature tall fescue than in the mature forage. Ferulic and protocatechuic acid levels did not differ between forages. 
Table 3. Expt 1. Digestibilities (proportion of intake digested) of dry matter, cell walls and lignin in various sections of the digestive tract of cannulated sheep

\begin{tabular}{|c|c|c|c|c|c|}
\hline \multirow[b]{2}{*}{$\begin{array}{l}\text { Diet... } \\
\text { Site of } \\
\text { digestion }\end{array}$} & \multicolumn{5}{|c|}{ Digestibility } \\
\hline & IML & ML & IMTF & MTF & SEM \\
\hline \multicolumn{6}{|c|}{ Dry matter } \\
\hline Total & 0.582 & 0.490 & 0.518 & 0.530 & 0.021 \\
\hline STO & 0.483 & 0.355 & 0.403 & 0.379 & 0.032 \\
\hline SI & 0.077 & 0.062 & 0.033 & 0.082 & 0.015 \\
\hline LI & 0.022 & 0.074 & 0.082 & 0.069 & 0.015 \\
\hline \multicolumn{6}{|l|}{ Cell walls } \\
\hline Total & 0.508 & 0.421 & 0.444 & 0.476 & 0.018 \\
\hline STO & 0.447 & $0 \cdot 316$ & 0.364 & 0.346 & 0.039 \\
\hline SI & 0.026 & 0.025 & -0.030 & $0 \cdot 043$ & 0.019 \\
\hline LI & 0.035 & 0.080 & $0 \cdot 110$ & 0.087 & 0.026 \\
\hline \multicolumn{6}{|l|}{ Lignin } \\
\hline Total & $0 \cdot 220$ & $0 \cdot 199$ & 0.183 & 0.129 & 0.046 \\
\hline STO & 0.208 & $0 \cdot 175$ & 0.220 & -0.024 & 0.067 \\
\hline SI & -0.008 & -0.090 & -0.172 & -0.050 & 0.093 \\
\hline LI & 0.019 & $0 \cdot 114$ & 0.135 & $0 \cdot 204$ & 0.118 \\
\hline
\end{tabular}

IML, immature lucerne (Medicago sativa) hay; ML, mature lucerne hay; IMTF, immature tall fescue (Festuca arundinacea) hay; MTF, mature tall fescue hay; Total, total tract; STO, reticulo-rumen, omasum and abomasum; SI, small intestine; LI, caecum and colon.

\section{Digestion of hay lignin and phenolic monomers}

During the adaptation phase of the third period of Expt 1, one of the sheep was lost from the study for reasons unrelated to treatment. Therefore, one observation each from the mature lucerne and immature tall fescue treatments was lost. Treatment means were calculated by the least squares method. Faecal recovery of the external marker was 0.90 (SE 0.03); therefore, calculated digestion coefficients are underestimates of apparent digestibility.

Digestibilities of DM, cell walls and lignin in the total digestive tract as well as in the various segments of the digestive tract are presented in Table 3. No significant differences between diets were found. Positive total tract digestibilities of lignin occurred and negative digestion coefficients for lignin were noted in the small intestine, regardless of diet (Table 3).

Apparent digestibilities of the alkali-labile phenolic monomers are given in Table 4. Greater $(P<0.05)$ total tract digestion of $p$-coumaric and ferulic acids of immature lucerne hay was observed as compared with the other hays, with mature tall fescue resulting in the lowest values. No differences were observed in total tract digestion of the other compounds. The same pattern was noted for digestion of $p$-coumaric and ferulic acids in the stomach (reticulo-rumen, omasum and abomasum). Sheep given mature lucerne hay had lower $(P<0.05)$ stomach digestion coefficients of $p$-hydroxybenzoic and protocatechuic acids than did sheep given the other diets (Table 4). Lower $(P<0.05)$ small intestine digestibilities were observed for $p$-coumaric, ferulic and protocatechuic acids in immature tall fescue hay compared with the other diets. Large intestine digestion of phenolic monomers did not differ 
Table 4. Expt 1. Apparent digestibilities (proportion of intake digested) of alkali-labile phenolic monomers in various sections of the digestive tract of cannulated sheep

\begin{tabular}{|c|c|c|c|c|c|}
\hline \multirow[b]{2}{*}{$\begin{array}{l}\text { Diet... } \\
\text { Phenolic site of } \\
\text { digestion }\end{array}$} & \multicolumn{5}{|c|}{ Digestibility } \\
\hline & IML & ML & IMTF & MTF & SEM \\
\hline \multicolumn{6}{|l|}{$p$-Coumaric acid } \\
\hline Total & $0.873^{\alpha}$ & $0.584^{b}$ & $0 \cdot 541^{b}$ & $0.391^{c}$ & 0.018 \\
\hline sTo & $0.801^{a}$ & $0.471^{b}$ & $0 \cdot 510^{b}$ & $0.296^{c}$ & 0.038 \\
\hline SI & $0.029^{a}$ & $0.117^{a}$ & $-0 \cdot 150^{b}$ & $0.072^{a}$ & 0.038 \\
\hline LI & 0.043 & -0.003 & $0 \cdot 181$ & 0.023 & 0.058 \\
\hline \multicolumn{6}{|l|}{ Ferulic acid } \\
\hline Total & $0.956^{a}$ & $0.829^{b}$ & $0.777^{c}$ & $0.651^{d}$ & 0.008 \\
\hline STO & $0 \cdot 914^{a}$ & $0.769^{b}$ & $0.749^{b}$ & $0.562^{c}$ & 0.024 \\
\hline SI & $0.014^{a}$ & $-0.026^{a}$ & $-0 \cdot 113^{b}$ & $0.020^{\alpha}$ & 0.015 \\
\hline LI & 0.028 & 0.086 & $0 \cdot 141$ & $0 \cdot 070$ & 0.032 \\
\hline \multicolumn{6}{|c|}{ p-Hydroxybenzoic acid } \\
\hline Total & 0.666 & 0.517 & 0.658 & $0 \cdot 553$ & 0.066 \\
\hline STO & $0 \cdot 631^{a}$ & $0.278^{b}$ & $0.710^{a}$ & $0.837^{a}$ & 0.086 \\
\hline SI & $0 \cdot 056$ & $0 \cdot 072$ & $-0 \cdot 123$ & $-0 \cdot 229$ & $0 \cdot 157$ \\
\hline LI & -0.021 & $0 \cdot 168$ & 0.072 & -0.054 & $0 \cdot 110$ \\
\hline \multicolumn{6}{|c|}{$p$-Hydroxybenzaldehyde } \\
\hline Total & $0 \cdot 346$ & $0 \cdot 142$ & 0.425 & $0 \cdot 191$ & 0.084 \\
\hline STO & 0.039 & -0.461 & 0.069 & -0.299 & $0 \cdot 169$ \\
\hline SI & $0 \cdot 166$ & 0.366 & 0.072 & 0.369 & 0.162 \\
\hline LI & $0 \cdot 141$ & 0.236 & 0.285 & 0.122 & 0.173 \\
\hline \multicolumn{6}{|c|}{ Protocatechuic acid } \\
\hline Total & 0.745 & 0.785 & 0.766 & 0.511 & 0.138 \\
\hline STO & $0.573^{a}$ & $0 \cdot 284^{b}$ & $0 \cdot 751^{a}$ & $0.350^{b}$ & 0.045 \\
\hline SI & $0 \cdot 209^{a}$ & $0 \cdot 242^{a}$ & $-0.037^{b}$ & $0 \cdot 328^{\alpha}$ & 0.057 \\
\hline LI & -0.037 & 0.259 & 0.052 & $-0 \cdot 167$ & $0 \cdot 160$ \\
\hline \multicolumn{6}{|l|}{ Vanillic acid } \\
\hline Total & 1.004 & 0.979 & 0.673 & 0.564 & 0.148 \\
\hline STO & 1.054 & 0.977 & 0.969 & 0.891 & 0.043 \\
\hline SI & -0.076 & -0.086 & -0.818 & -0.182 & $0 \cdot 153$ \\
\hline LI & $0.026^{A}$ & $0.088^{A}$ & $0.522^{B}$ & $-0 \cdot 146^{A}$ & 0.118 \\
\hline \multicolumn{6}{|l|}{ Vanillin } \\
\hline Total & 0.261 & $0-502$ & 0.122 & 0.443 & 0.279 \\
\hline STO & -0.365 & -0.255 & -0.377 & 0.283 & 0.707 \\
\hline SI & 0.407 & 0.461 & 0.078 & 0.168 & 0.245 \\
\hline LI & 0.219 & 0.295 & 0.421 & 0.007 & 0.229 \\
\hline
\end{tabular}

IML, immature lucerne (Medicago sativa) hay; ML, mature lucerne hay; IMTF, immature tall fescue (Festuca arundinacea) hay; MTF, mature tall fescue hay; Total, total tract; STO, reticulo-rumen, omasum and abomasum; SI, small intestine; LI, caecum and colon.

$a, b, c, a$ Mean values in the same row not sharing common superscript letters differed significantly $(P<0 \cdot 05)$. $A, B$ Mean values in the same row not sharing common superscript letters differed significantly $(P<0 \cdot 10)$.

between diets (Table 4), except for vanillic acid which was more digestible $(P<0 \cdot 10)$ in immature tall fescue hay.

In Table 5, the apparent digestibilities of the nitrobenzene oxidation products of lignin are summarized. They were characterized by negative digestion coefficients and high variances. Significant differences were noted in some of the nitrobenzene oxidation products of dietary lignin and the lignin recovered from the digesta. These are presented in Table 6. 
Table 5. Expt 1. Apparent digestibilities (proportion of intake digested) of nitrobenzene oxidation products in various sections of the digestive tract of cannulated sheep

\begin{tabular}{|c|c|c|c|c|c|}
\hline \multirow[b]{2}{*}{$\begin{array}{l}\text { Diet... } \\
\text { Phenolic site of } \\
\text { digestion }\end{array}$} & \multicolumn{5}{|c|}{ Digestibility } \\
\hline & IML & ML & IMTF & MTF & SEM \\
\hline \multicolumn{6}{|l|}{$p$-Coumaric acid } \\
\hline Total & $0 \cdot 139^{a}$ & $0.065^{a}$ & $-0.026^{a}$ & $0.595^{b}$ & $0 \cdot 105$ \\
\hline STO & 0.215 & 0.335 & 0.064 & 0.472 & 0.071 \\
\hline SI & -0.252 & $-0 \cdot 240$ & -0.279 & 0.014 & $0 \cdot 173$ \\
\hline LI & 0.176 & -0.030 & $0 \cdot 189$ & $0 \cdot 109$ & 0.240 \\
\hline \multicolumn{6}{|l|}{ Ferulic acid } \\
\hline Total & -0.411 & $-1 \cdot 137$ & -0.079 & 0.527 & 0.726 \\
\hline STO & $-2 \cdot 309 A$ & $0 \cdot 108^{B}$ & $0.619^{B}$ & $0 \cdot 566^{B}$ & 0.668 \\
\hline SI & 2.086 & -2.758 & -2.530 & -0.526 & $1 \cdot 192$ \\
\hline LI & $-0 \cdot 188$ & 1.513 & 1.832 & 0.487 & 0.874 \\
\hline \multicolumn{6}{|c|}{$p$-Hydroxybenzaldehyde } \\
\hline Total & $0.081^{a}$ & $-0.033^{a b}$ & $-0.402^{b}$ & $0 \cdot 336^{a}$ & 0.099 \\
\hline sTo & 0.132 & 0.218 & $0 \cdot 107$ & 0.273 & 0.128 \\
\hline SI & 0.016 & $-0 \cdot 170$ & -0.357 & -0.304 & $0 \cdot 100$ \\
\hline LI & $-0.066^{A}$ & $-0.081^{A}$ & $-0 \cdot 152^{A}$ & $0 \cdot 367^{B}$ & 0.101 \\
\hline \multicolumn{6}{|c|}{ Protocatechuic acid } \\
\hline Total & 0.560 & 0.244 & -0.349 & 0.585 & 0.316 \\
\hline STO & $0.569^{A}$ & $0.433^{A}$ & $-0 \cdot 183^{B}$ & $0.631^{A}$ & 0.147 \\
\hline SI & 0.026 & -0.091 & $-0 \cdot 105$ & -0.050 & $0 \cdot 120$ \\
\hline LI & -0.035 & $-0 \cdot 099$ & -0.061 & 0.004 & 0.213 \\
\hline \multicolumn{6}{|l|}{ Syringic acid } \\
\hline Total & $-0.966^{a}$ & $0 \cdot 701^{b}$ & $0.847^{b}$ & $0.868^{b}$ & 0.274 \\
\hline STO & $-2.851^{a}$ & $1 \cdot 276^{b}$ & $1 \cdot 320^{b}$ & $0.729^{b}$ & 0.603 \\
\hline SI & $2 \cdot 198^{a}$ & $-0.603^{b}$ & $-0.256^{b}$ & $-0.043^{b}$ & 0.460 \\
\hline LI & -0.343 & 0.028 & -0.216 & $0 \cdot 182$ & 0.600 \\
\hline \multicolumn{6}{|l|}{ Syringaldehyde } \\
\hline Total & $0.020^{a}$ & $0.008^{a}$ & $0 \cdot 185^{a}$ & $0.527^{\circ}$ & 0.065 \\
\hline STO & $-0 \cdot 160$ & 0.114 & $0 \cdot 165$ & 0.319 & 0.139 \\
\hline SI & 0.290 & -0.030 & 0.167 & 0.057 & $0 \cdot 173$ \\
\hline LI & $-0 \cdot 110^{4}$ & $-0.077^{A}$ & $-0 \cdot 147^{A}$ & $0 \cdot 151^{B}$ & 0.062 \\
\hline \multicolumn{6}{|l|}{ Vanillic acid } \\
\hline Total & $0 \cdot 185^{A}$ & $0 \cdot 152^{A}$ & $-0 \cdot 314^{B}$ & $0.543^{A}$ & $0 \cdot 141$ \\
\hline STO & $0 \cdot 151$ & $0 \cdot 198$ & -0.066 & $0 \cdot 410$ & $0 \cdot 143$ \\
\hline SI & $0 \cdot 172$ & -0.019 & -0.135 & -0.093 & 0.095 \\
\hline $\mathbf{L I}$ & $-0 \cdot 138$ & -0.027 & 0.113 & 0.226 & $0 \cdot 101$ \\
\hline \multicolumn{6}{|l|}{ Vanillin } \\
\hline Total & $-0 \cdot 285^{a}$ & $-0 \cdot 363^{a}$ & $-0.305^{a}$ & $0.411^{b}$ & 0.074 \\
\hline STO & -0.314 & -0.001 & -0.050 & $0 \cdot 189$ & $0 \cdot 140$ \\
\hline SI & $0 \cdot 160$ & -0.212 & 0.021 & $-0 \cdot 105$ & 0.212 \\
\hline LI & $-0 \cdot 132^{a}$ & $-0 \cdot 149^{a}$ & $-0.275^{a}$ & $0 \cdot 326^{b}$ & 0.077 \\
\hline
\end{tabular}

IML, immature lucerne (Medicago sativa) hay; ML, mature lucerne hay; IMTF, immature tall fescue (Festuca arundinacea) hay; MTF, mature tall fescue hay; Total, total tract; STO, reticulo-rumen, omasum and abomasum; SI, small intestine; LI, caecum and colon.

$a, b$ Mean values in the same row not sharing common superscript letters differed significantly $(P<0.05)$.

$A, B$ Mean values in the same row not sharing common superscript letters differed significantly $(P<0 \cdot 10)$. 


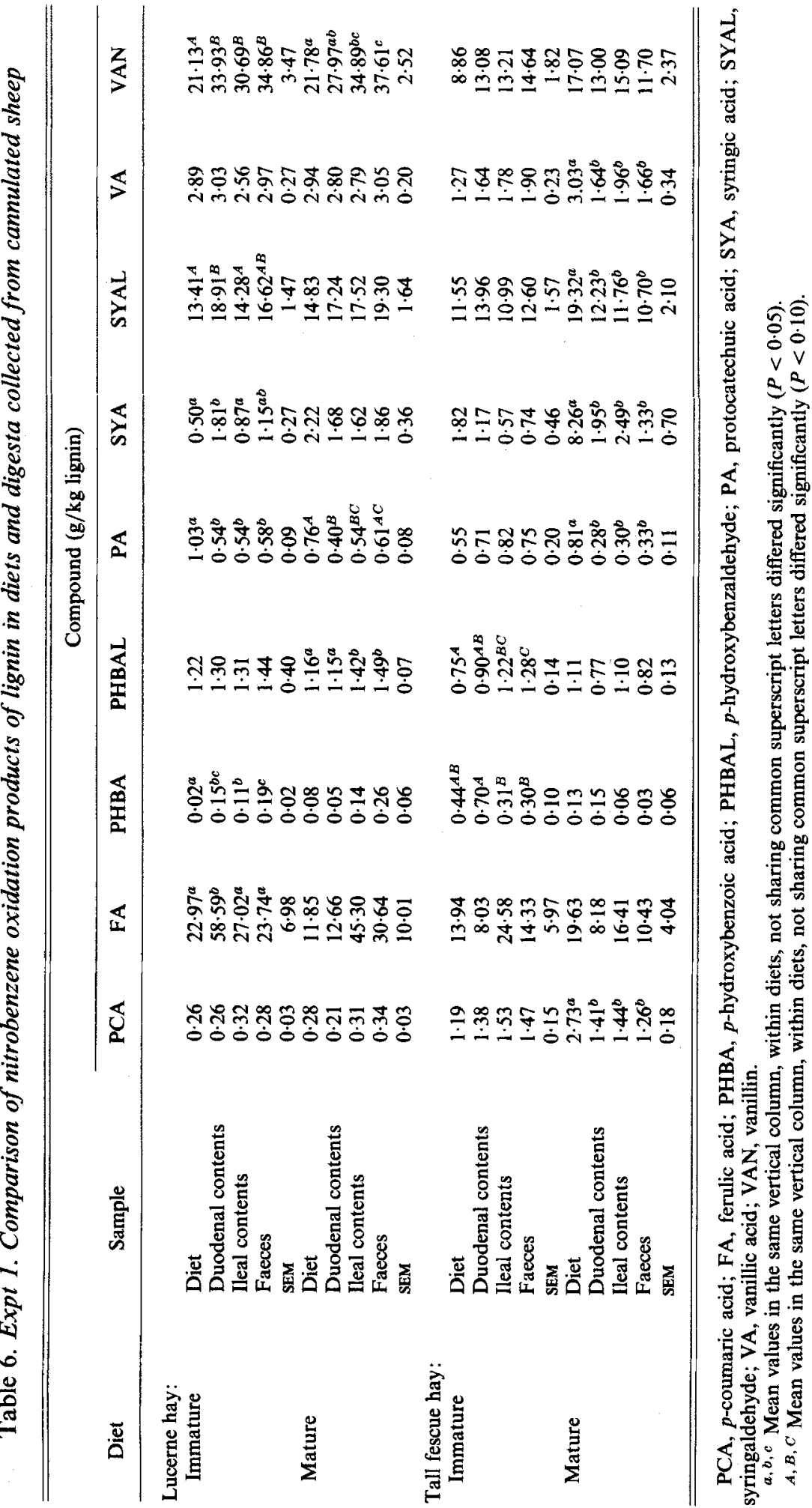


Table 7. Expt 1. Intake ( $m g / d)$, faecal excretion (FE), urinary excretion (UE) and non-recovery (NR) (proportion of intake) of phenolic monomers by sheep

\begin{tabular}{|c|c|c|c|c|c|}
\hline \multirow[b]{2}{*}{ Phenolic } & \multicolumn{5}{|c|}{ Diet } \\
\hline & IML & ML & IMTF & MTF & SEM \\
\hline \multicolumn{6}{|c|}{ p-Coumaric acid } \\
\hline Intake & $453 \cdot 7^{a}$ & $522 \cdot 6^{a}$ & $1356 \cdot 9^{b}$ & $2420 \cdot 3^{c}$ & $109 \cdot 0$ \\
\hline FE & $0 \cdot 134^{a}$ & $0.401^{b}$ & $0.410^{b}$ & $0.577^{c}$ & 0.031 \\
\hline UE & $0.013^{A C}$ & $0.027^{B}$ & $0.019^{A B}$ & $0.005^{C}$ & 0.004 \\
\hline NR & $0.854^{a}$ & $0.573^{b}$ & $0.571^{b}$ & $0.418^{c}$ & 0.029 \\
\hline \multicolumn{6}{|c|}{ Ferulic acid } \\
\hline Intake & $2264 \cdot 0$ & $2037 \cdot 6$ & $2124 \cdot 9$ & $2485 \cdot 5$ & $334 \cdot 4$ \\
\hline $\mathrm{FE}$ & 0.537 & $1 \cdot 201$ & 0.127 & 0.361 & 0.271 \\
\hline UE & 0.000 & 0.002 & 0.002 & 0.001 & 0.001 \\
\hline NR & 0.463 & -0.201 & 0.871 & 0.638 & 0.271 \\
\hline \multicolumn{6}{|c|}{ p-Hydroxybenzoic acid } \\
\hline Intake & 30.9 & $47 \cdot 0$ & $47 \cdot 3$ & $33 \cdot 2$ & $4 \cdot 7$ \\
\hline $\mathrm{FE}$ & $0.520^{A B}$ & $0 \cdot 804^{A}$ & $0.341^{B}$ & $0.414^{B}$ & 0.095 \\
\hline UE & 0.658 & 0.206 & $0 \cdot 123$ & 0.386 & 0.244 \\
\hline NR & -0.178 & -0.010 & 0.536 & $0 \cdot 201$ & 0.189 \\
\hline \multicolumn{6}{|c|}{$p$-Hydroxybenzaldehyde } \\
\hline Intake & $89 \cdot 1^{\alpha}$ & $119 \cdot 7^{b}$ & $66 \cdot 5^{c}$ & $81 \cdot 4^{a c}$ & $4 \cdot 1$ \\
\hline $\mathrm{FE}$ & $0 \cdot 764^{a}$ & $0.909^{b}$ & $0.780^{a}$ & $0.696^{a}$ & 0.030 \\
\hline UE & $0 \cdot 180$ & 0.063 & 0.016 & 0.043 & 0.081 \\
\hline NR & 0.057 & 0.028 & 0.204 & $0 \cdot 261$ & 0.098 \\
\hline \multicolumn{6}{|c|}{ Protocatechuic acid } \\
\hline Intake & $92 \cdot 2^{a}$ & $88 \cdot 3^{a}$ & $67 \cdot 2^{b}$ & $51 \cdot 4^{b}$ & 3.9 \\
\hline FE & 0.331 & 0.458 & 0.421 & 0.420 & 0.119 \\
\hline UE & $0 \cdot 132$ & $0 \cdot 070$ & 0.067 & 0.077 & 0.048 \\
\hline NR & 0.537 & 0.472 & 0.512 & 0.503 & $0 \cdot 108$ \\
\hline \multicolumn{6}{|c|}{ Syringic acid } \\
\hline Intake & $32 \cdot 9^{a}$ & $187 \cdot 7^{b}$ & $48 \cdot 3^{a}$ & $263 \cdot 1^{b}$ & $28 \cdot 3$ \\
\hline FE & $1 \cdot 692^{a}$ & $0 \cdot 366^{b}$ & $0 \cdot 118^{b}$ & $0 \cdot 126^{b}$ & 0.279 \\
\hline UE & 0.322 & 0.001 & 0.037 & 0.005 & 0.139 \\
\hline NR & $-1 \cdot 014^{a}$ & $0.633^{b}$ & $0.845^{b}$ & $0.870^{b}$ & 0.367 \\
\hline \multicolumn{6}{|c|}{ Syringaldehyde } \\
\hline Intake & $873 \cdot 1^{a}$ & $1307 \cdot 8^{b}$ & $438 \cdot 5^{c}$ & $621 \cdot 6^{c}$ & $67 \cdot 8$ \\
\hline FE & $0.841^{a}$ & $0.944^{a}$ & $0.702^{a b}$ & $0.463^{b}$ & 0.076 \\
\hline UE & 0.000 & 0.000 & 0.000 & 0.000 & - \\
\hline NR & $0 \cdot 159^{a}$ & $0.056^{a}$ & $0 \cdot 298^{a b}$ & $0.537^{b}$ & 0.076 \\
\hline \multicolumn{6}{|c|}{ Vanillic acid } \\
\hline Intake & $189 \cdot 6^{a}$ & $269 \cdot 6^{b}$ & $74 \cdot 0^{c}$ & $139 \cdot 8^{d}$ & 12.9 \\
\hline $\mathrm{FE}$ & $0.670^{A}$ & $0.716^{A}$ & $0.740^{A}$ & $0.433^{B}$ & 0.069 \\
\hline UE & 0.000 & 0.000 & 0.006 & 0.018 & 0.010 \\
\hline NR & $0 \cdot 330^{A}$ & $0 \cdot 284^{A}$ & $0.255^{A}$ & $0.549^{B}$ & 0.065 \\
\hline \multicolumn{6}{|l|}{ Vanillin } \\
\hline Intake & $1381 \cdot 1^{a}$ & $1960 \cdot 8^{b}$ & $484 \cdot 2^{c}$ & $647 \cdot 8^{c}$ & 116.4 \\
\hline $\mathrm{FE}$ & $1 \cdot 103^{a b}$ & $1 \cdot 230^{b}$ & $0.951^{a}$ & $0.574^{c}$ & 0.051 \\
\hline UE & 0.000 & 0.000 & 0.000 & 0.000 & - \\
\hline NR & $-0 \cdot 103^{a b}$ & $-0 \cdot 230^{b}$ & $0 \cdot 049^{a}$ & $0.427^{c}$ & 0.051 \\
\hline \multicolumn{6}{|c|}{ Total phenolics } \\
\hline Intake & $5406 \cdot 5$ & $6541 \cdot 0$ & $4708 \cdot 1$ & 6743.9 & $404 \cdot 7$ \\
\hline $\mathrm{FE}$ & $0 \cdot 698^{a}$ & $1.004^{b}$ & $0.367^{c}$ & $0.465^{c}$ & 0.063 \\
\hline UE & 0.014 & 0.005 & 0.010 & 0.006 & 0.004 \\
\hline NR & $0.288^{a}$ & $-0.009^{b}$ & $0.623^{c}$ & $0.529^{c}$ & 0.059 \\
\hline
\end{tabular}

IML, immature lucerne (Medicago sativa) hay; ML, mature lucerne hay; IMTF, immature tall fescue (Festuca arundinacea) hay; MTF, mature tall fescue hay.

$a, b, c$ Mean values in the same row not sharing common superscript letters differed significantly $(P<0.05)$.

$A, B, C$ Mean values in the same row not sharing common superscript letters differed significantly $(P<0 \cdot 10)$. 


\section{Recovery of phenolic monomers in excreta}

Phenolic monomer balances are presented in Table 7. Both alkali-labile and nitrobenzene oxidation phenolic monomers are included. Intakes of protocatechuic acid, syringaldehyde, vanillic acid and vanillin were greater $(P<0.05)$ for legume hays than for grass hays. $p$-Hydroxybenzaldehyde and syringic acid intakes were significantly greater when sheep were given mature lucerne and tall fescue diets than when given their immature counterparts. Intake of ferulic acid, $p$-hydroxybenzoic acid and total phenolics did not differ between diets.

Faecal and urinary excretions of phenolics are expressed as a percentage of intake (Table 7). Faecal excretion of $p$-coumaric acid by sheep given immature lucerne was lower $(P<0.05)$ than for sheep given the other diets and was greatest when animals were given mature tall fescue. Feeding mature lucerne hay resulted in elevated faecal excretion of $p$-hydroxybenzoic acid and $p$-hydroxybenzaldehyde (Table 7). Faecal losses of syringic acid for sheep given immature lucerne were significantly greater than when sheep were given the other diets. Feeding the two grass hays resulted in lower faecal excretion of syringaldehyde, vanillin and total phenolics compared with results obtained from feeding the legume diets (Table 7). Vanillic acid loss in faeces was also reduced $(P<0 \cdot 10)$ for animals given the mature tall fescue diet. Urinary excretion was very low and did not differ between diets for either individual phenolic monomers or total phenolics, except for $p$-coumaric acid which was excreted to a greater $(P<0 \cdot 10)$ extent by sheep given mature lucerne hays as compared with immature lucerne or mature tall fescue. Sheep given immature lucerne had a higher $(P<0.05)$ proportion of non-recovered $p$-coumaric acid and lower $(P<0.05)$ non-recovery of syringic acid than animals given the other diets (Table 7). Non-recovery of vanillin was lower $(P<0.05)$ for sheep given the mature lucerne diet. A significantly lower proportion of non-recovered $p$-coumaric acid and greater $(P<0.05)$ non-recovery of syringaldehyde, vanillic acid and vanillin were observed for sheep given mature tall fescue hay. Over-all, non-recovery of total phenolic monomers were greater $(P<0.05)$ for sheep consuming fescue hays than for those consuming lucerne hays (Table 7).

\section{Concentrations of soluble phenolic monomers}

Concentrations of soluble phenolic monomers in the liquid phase of digesta collected from the rumen, duodenum and ileum are given in Table 8. Concentrations were quite low and few differences between diets existed. Sheep given the grass hays had greater $(P<0.05)$ rumen and ileal concentrations of $p$-coumaric acid than animals given lucerne hays. Ferulic acid concentrations were greater $(P<0 \cdot 10)$ in rumen fluid of sheep given immature tall fescue hay. Rumen concentrations of $p$-hydroxybenzaldehyde were lower for animals given immature lucerne hay than for those given the other diets (Table 8). Duodenal levels of this compound were greater $(P<0 \cdot 10)$ in sheep given the immature tall fescue diet. Protocatechuic acid concentrations in the duodenum of animals consuming immature lucerne were greater $(P<0.05)$ than for the other diets. The most obvious differences in soluble phenolics between samples were the consistently lower concentrations in duodenal contents, compared with rumen and ileal contents, for all diets and compounds (Table 9).

Results from Expt 2 are given in Table 9. Concentrations of soluble phenolic monomers were greater $(P<0.05)$ in rumen fluid than in abomasal contents of the steer. Rumen levels of all phenolics declined $(P<0.05)$ from 0 to $4 \mathrm{~h}$ post-feeding. $p$-Coumaric and vanillic acids reached a secondary peak in concentration in the rumen at $12 \mathrm{~h}$ post-feeding. Abomasal levels of phenolic monomers did not change significantly with time after feeding (Table 9) and were always low relative to rumen concentrations. 
Table 8. Expt 1. Soluble phenolic monomers in digesta collected at various sites in the gastrointestinal tract of cannulated sheep

\begin{tabular}{|c|c|c|c|c|c|c|}
\hline \multirow[b]{2}{*}{$\begin{array}{l}\text { Diet... } \\
\text { Phenolic }\end{array}$} & \multirow[b]{2}{*}{ Sampling site } & \multicolumn{5}{|c|}{ Concentration (mg/l) } \\
\hline & & IML & ML & IMTF & MTF & SEM \\
\hline$p$-Coumaric acid & $\begin{array}{l}\text { Rumen } \\
\text { Duodenum } \\
\text { Ileum }\end{array}$ & $\begin{array}{l}0 \cdot 18^{a} \\
0 \cdot 03 \\
1.90^{a}\end{array}$ & $\begin{array}{l}0 \cdot 20^{a} \\
0 \cdot 00 \\
2 \cdot 05^{a}\end{array}$ & $\begin{array}{l}0 \cdot 83^{b} \\
0 \cdot 02 \\
6 \cdot 38^{b}\end{array}$ & $\begin{array}{l}1 \cdot 30^{b} \\
0 \cdot 00 \\
8 \cdot 78^{b}\end{array}$ & $\begin{array}{l}0.12 \\
0.02 \\
0.63\end{array}$ \\
\hline Ferulic acid & $\begin{array}{l}\text { Rumen } \\
\text { Duodenum } \\
\text { Ileum }\end{array}$ & $\begin{array}{l}0.08^{A} \\
0 \cdot 00 \\
3.88\end{array}$ & $\begin{array}{l}0.03^{A} \\
0.00 \\
1.23\end{array}$ & $\begin{array}{l}1 \cdot 00^{B} \\
0 \cdot 00 \\
2.49\end{array}$ & $\begin{array}{l}0 \cdot 20^{4} \\
0 \cdot 00 \\
1.43\end{array}$ & $\frac{0 \cdot 18}{1 \cdot 12}$ \\
\hline$p$-Hydroxybenzoic acid & $\begin{array}{l}\text { Rumen } \\
\text { Duodenum } \\
\text { Ileum }\end{array}$ & $\begin{array}{l}0.98 \\
0.13 \\
1.65\end{array}$ & $\begin{array}{l}0.81 \\
0.00 \\
2.08\end{array}$ & $\begin{array}{l}0.21 \\
0.00 \\
1.87\end{array}$ & $\begin{array}{l}0.55 \\
0.00 \\
2 \cdot 03\end{array}$ & $\begin{array}{l}0.27 \\
0.04 \\
0.59\end{array}$ \\
\hline$p$-Hydroxybenzaldehyde & $\begin{array}{l}\text { Rumen } \\
\text { Duodenum } \\
\text { Ileum }\end{array}$ & $\begin{array}{l}0.20^{A} \\
0.03^{A B} \\
0.48\end{array}$ & $\begin{array}{l}0.28^{B} \\
0.00^{A} \\
0.63\end{array}$ & $\begin{array}{l}0 \cdot 32^{B} \\
0.07^{B} \\
0.78\end{array}$ & $\begin{array}{l}0.33^{B} \\
0.00^{A} \\
0.73\end{array}$ & $\begin{array}{l}0.03 \\
0.02 \\
0.10\end{array}$ \\
\hline Protocatechuic acid & $\begin{array}{l}\text { Rumen } \\
\text { Duodenum } \\
\text { Ileum }\end{array}$ & $\begin{array}{l}1 \cdot 40 \\
0 \cdot 45^{a} \\
1 \cdot 50\end{array}$ & $\begin{array}{l}1 \cdot 00 \\
0 \cdot 06^{b} \\
0 \cdot 84\end{array}$ & $\begin{array}{l}0.98 \\
0 \cdot 26^{a b} \\
1 \cdot 18\end{array}$ & $\begin{array}{c}0.88 \\
0 \cdot 18^{b} \\
0 \cdot 80\end{array}$ & $\begin{array}{l}0.20 \\
0.06 \\
0.23\end{array}$ \\
\hline Syringic acid & $\begin{array}{l}\text { Rumen } \\
\text { Duodenum } \\
\text { Ileum }\end{array}$ & $\begin{array}{l}0.00 \\
0.00 \\
0.00\end{array}$ & $\begin{array}{l}0.08 \\
0.00 \\
0.20\end{array}$ & $\begin{array}{l}0.08 \\
0.00 \\
0.00\end{array}$ & $\begin{array}{l}0.15 \\
0.00 \\
3 \cdot 20\end{array}$ & $\frac{0.09}{1.37}$ \\
\hline Syringaldehyde & $\begin{array}{l}\text { Rumen } \\
\text { Duodenum } \\
\text { Ileum }\end{array}$ & $\begin{array}{l}0.00 \\
0.00 \\
0.00\end{array}$ & $\begin{array}{l}0.04 \\
0.00 \\
0.04\end{array}$ & $\begin{array}{l}0.04 \\
0.06 \\
0 \cdot 11\end{array}$ & $\begin{array}{l}0.08 \\
0.00 \\
0.12\end{array}$ & $\begin{array}{l}0.04 \\
0.03 \\
0.07\end{array}$ \\
\hline Vanillic acid & $\begin{array}{l}\text { Rumen } \\
\text { Duodenum } \\
\text { Ileum }\end{array}$ & $\begin{array}{l}0.58 \\
0.03 \\
0.53\end{array}$ & $\begin{array}{l}0.21 \\
0.01 \\
0.67\end{array}$ & $\begin{array}{l}0.11 \\
0.01 \\
1.63\end{array}$ & $\begin{array}{l}0.10 \\
0.00 \\
1 \cdot 25\end{array}$ & $\begin{array}{l}0.45 \\
0.02 \\
0.33\end{array}$ \\
\hline Vanillin & $\begin{array}{l}\text { Rumen } \\
\text { Duodenum } \\
\text { Ileum }\end{array}$ & $\begin{array}{l}0 \cdot 38 \\
0 \cdot 00 \\
0-43\end{array}$ & $\begin{array}{l}1.01 \\
0.01 \\
0.46\end{array}$ & $\begin{array}{l}0.46 \\
0.03 \\
0.94\end{array}$ & $\begin{array}{l}0.20 \\
0.00 \\
0.83\end{array}$ & $\begin{array}{l}0.27 \\
0.02 \\
0.13\end{array}$ \\
\hline
\end{tabular}

IML, immature lucerne (Medicago sativa) hay; ML, mature lucerne hay; IMTF, immature tall fescue (Festuca arundinacea) hay; MTF, mature tall fescue hay.

${ }^{a, b}$ Mean values in the same row not sharing common superscript letters differed significantly $(P<0.05)$.

${ }^{A, B}$ Mean values in the same row not sharing common superscript letters differed significantly $(P<0 \cdot 10)$.

\section{DISCUSSION}

Grasses have been reported to contain higher concentrations of alkali-labile phenolic monomers in their cell walls than legumes (Hartley \& Jones, 1977; Jung et al. 1983). This conclusion is supported by values for the grass and legume hays analysed in this study.

The higher concentrations of $p$-coumaric and ferulic acids seen for timothy (Phleum pratense) as physiological maturity advanced (Theander et al. 1981) was also found for tall fescue in the present work. However, maturation did not result in a similar effect for lucerne. This suggests a basic difference between grasses and legumes in deposition of phenolic monomers, as the lignin composition (nitrobenzene product formation) of tall fescue also was altered during maturation whereas that of the lucerne was constant. These differences may relate to the apparently greater inhibitory effects of grass lignin than of legume lignin on cell wall digestibility. 
Table 9. Expt 2. Concentrations of soluble phenolic monomers in rumen and abomasal fluids collected at various times post-feeding from a cannulated steer given immature lucerne (Medicago sativa) hay

\begin{tabular}{|c|c|c|c|c|c|c|}
\hline \multirow[b]{2}{*}{ Site } & \multirow{2}{*}{$\begin{array}{c}\text { Period } \\
\text { post-feeding (h) }\end{array}$} & \multicolumn{5}{|c|}{ Compound (mg/l) } \\
\hline & & PCA & PHBA & PHBAL & PA & VA \\
\hline \multirow[t]{5}{*}{ Rumen } & 0 & $0.57^{a}$ & $1 \cdot 21^{a}$ & $0 \cdot 22^{a}$ & $8 \cdot 35^{a}$ & $3.64^{\circ}$ \\
\hline & 4 & $0.06^{b}$ & $0 \cdot 13^{b}$ & $0.07^{b}$ & $2.89^{b}$ & $0.22^{b}$ \\
\hline & 8 & $0.12^{b c}$ & $0.15^{b}$ & $0 \cdot 12^{b}$ & $1 \cdot 19^{b}$ & $0.51^{t}$ \\
\hline & 12 & $0 \cdot 24^{c}$ & $0 \cdot 27^{b}$ & $0.09^{b}$ & $0.94^{b}$ & $1.41^{\circ}$ \\
\hline & 24 & $0 \cdot 17^{b c}$ & $0 \cdot 24^{b}$ & $0.10^{\circ}$ & $0.93^{b}$ & $0.54^{t}$ \\
\hline SEM & & 0.05 & $0 \cdot 14$ & 0.02 & 0.95 & 0.20 \\
\hline \multirow{5}{*}{ Abomasum } & 0 & 0.02 & 0.08 & 0.03 & 0.49 & 0.09 \\
\hline & 4 & 0.01 & 0.00 & 0.03 & 0.38 & 0.01 \\
\hline & 8 & 0.01 & 0.09 & 0.03 & 0.51 & 0.00 \\
\hline & 12 & 0.05 & $0 \cdot 10$ & 0.05 & 0.42 & 0.00 \\
\hline & 24 & 0.00 & 0.00 & 0.03 & 0.25 & 0.00 \\
\hline SEM & & 0.01 & 0.06 & 0.01 & 0.09 & 0.04 \\
\hline
\end{tabular}

PCA, $p$-coumaric acid; PHBA, $p$-hydroxybenzoic acid; PHBAL, $p$-hydroxybenzaldehyde; PA, protocatechuic acid; VA, vanillic acid.

$a, b, c$ Mean values in the same vertical column, within site, not sharing common superscript letters differed significantly $(P<0.05)$.

Interpretation of phenolic monomer composition and digestibility results is limited by the extraction procedures used. The nitrobenzene oxidation products only account for $40-70 \mathrm{~g}$ of phenolic monomers $/ \mathrm{kg}$ lignin. The remainder of the lignin is not extractable due to its chemical structure. Modification of lignin chemistry during digestion may alter the proportion of lignin extracted by a given method. Also, apparent digestion of phenolic monomers in sections of the gastrointestinal tract may represent solubilization rather than true digestion because only the digesta solids were analysed quantitatively. However, this problem is not encountered for total tract digestibility.

\section{Digestibility of lignin and phenolic monomers}

While lignin has often been reported to be indigestible (Crampton \& Maynard, 1938; Forbes et al. 1946; Kane et al. 1950), recent work indicates lignin is apparently digested (Allinson \& Osbourn, 1970; Fahey et al. 1979). The analytical technique used affects the digestibility estimate, although relative differences between diets are generally similar (Muntifering et al. 1981). The hays used in this study also resulted in positive apparent digestibilities, averaging 0.183 across all diets. The vast majority of this digestion occurred in the stomach, with only minor changes taking place in the intestines. Gaillard \& Richards (1975) reported the rumen to be the site of lignin digestion, via the production of soluble lignin-carbohydrate complexes. Lignin leaving the rumen underwent little further digestion in the intestinal tract (Neilson \& Richards, 1978). Rumen digestion of lignin appears to be partially a result of microbial activity, as Akin (1980) reported the isolation from the rumen of a bacterium which degraded lignified vascular tissue.

Digestion of the majority of phenolic monomers took place primarily in the rumen, although some compounds underwent substantial concentration changes in the intestines. Theander et al. (1981) found that rumen micro-organisms reduced the ferulic acid content of grass cell walls to a greater extent than the $p$-coumaric acid content of grass cell walls. 
This was also observed for a legume and two grass species by Jung et al. (1983). Ferulic acid in the legume was more digestible than ferulic acid in the grasses (Jung et al. 1983). Similar results were generally seen for total tract digestibility of alkali-labile phenolics by sheep given the lucerne and tall fescue hays used in the present experiments. The digestibilities of alkali-labile $p$-coumaric and ferulic acids were greater with lucerne diets than with tall fescue diets.

There are several potential explanations for the apparent digestibilities of these phenolic monomers. In the case of positive digestion coefficients, phenolic monomers may be fermented as a source of energy by rumen microbes or released as soluble phenolic glycosides. The former has been demonstrated to occur in vitro (Akin, 1980), although the slow growth rate of the rumen bacterium involved would presumably preclude its survival in the rumen using phenolics as an energy source. Production of soluble glycosides of ferulic acid after cellulase treatment of grass cell walls is also known to occur (Hartley et al. 1976). Negative digestion coefficients are more difficult to explain, but may represent either an increased extractability of phenolic monomers from plant material due to physico-chemical modifications during the digestive process or precipitation of soluble phenolics during passage through the gut. Neilson \& Richards (1978) reported precipitation of soluble lignin-carbohydrate complexes in the abomasum of steers. Also, forages contain significant quantities (approximately $30 \%$ ) of phenolic monomers in the cell-soluble fraction (Jung et al. 1983) which may bind to the digesta solids under appropriate conditions.

Not only was lignin digested by the sheep, but the composition of the remaining lignin was altered. Previous work showed that maize (Zea mays) plant lignin was modified by rumen digestion in cattle (Cymbaluk et al. 1973). Both normal and brown midrib (low lignin) maize were found to have decreased phenolic aldehyde contents and increased phenolic acid contents after suspension in the rumen (Cymbaluk et al. 1973). Lucerne and smooth bromegrass (Bromus inermis) lignins decreased in their concentrations of both phenolic acids and aldehydes in all sections of the gastrointestinal tract (Fahey et al. 1980). In Expt 1 of the present study, no consistent trend in digestion for either phenolic acids or aldehydes was noted for any of the diets.

\section{Metabolism of phenolic monomers}

Infusion of cinnamic acid derivatives into the rumen of sheep resulted in elevated urinary benzoic acid excretion, whereas abomasal infusion or infusion of benzoic acid derivatives at either site had no effect (Martin, 1982). Urine samples collected throughout the present study and subjected to high-pressure liquid chromatographic analysis had many large, unidentified peaks with short retention times, suggesting metabolism of forage phenolics to various derivatives which are generally more water-soluble than natural forage phenolics. Liquid digesta samples did not contain large amounts of these unidentified peaks, but incubation of phenolic monomers in vitro with rumen microbes produced chromatographs similar to those noted on analysis of sheep urine (Jung \& Fahey, 1983b). Urine from rats given phenolic monomers did not contain these peaks (Jung \& Fahey, 1983c). Apparently, microbial metabolism is involved in the production of these phenolic derivatives. Martin (1982) reported the urinary excretion of 3-phenylpropionic and benzoic acids by sheep given forages. Infusion of cinnamic acid derivatives into the rumen increased urinary excretion of these compounds (Martin, 1982). While these compounds were not analysed for directly, being aromatic carboxylic acids, 3-phenylpropionic and benzoic acids should have been present in the group of unidentified peaks in the samples from our study. These two compounds would not account for a significant proportion of the total apparent digestion of phenolic monomers by the sheep based on the amount of material represented by the chromatographs. Chesson et al. (1982) found no evidence of formation of 3-phenylpropionic 
acid by pure or mixed cultures of rumen bacteria in vitro. Vanillin does not appear to be excreted in the urine of mammals, as neither the urine of sheep used in the present study nor the urine of rats in previous work (Scheline, 1978; Jung \& Fahey, 1983c) has been found to contain vanillin, even though it was an important constituent of the diet and apparently absorbed.

Our results indicate significant lack of recovery of most phenolic monomers in sheep given the forages. There is no reason to assume that the animals sequester phenolics in their bodies and inclusion of the unidentified compounds from the urine in the total recovery of phenolics still results in significant under-recoveries. Therefore, the possibility of metabolism of the phenolic monomers to non-aromatic products must be considered. The aromatic amino acids, phenylalanine and tyrosine, are catabolized to fumarate and acetate by the mammalian liver (Rodwell, 1979), suggesting that the aromatic ring of phenolic monomers may be susceptible to oxidative cleavage by ruminants. Products such as fumarate and acetate can, of course, be utilized as sources of energy. While use of phenolic monomers as energy sources has not been demonstrated for ruminants, termites apparently absorb and oxidize phenolic monomers released from lignin by their symbiotic gut microflora (Butler \& Buckerfield, 1979).

\section{Fluctuation of soluble phenolic concentrations}

Neilson \& Richards (1978) reported that the soluble lignin-carbohydrate complexes produced in the rumen were precipitated by the acid conditions of the abomasum. The dramatic decrease in soluble phenolics in abomasal and duodenal digesta observed in our experiments, relative to rumen levels, supports the hypothesis that phenolics precipitate under acid conditions. Unlike previous reports (Neilson \& Richards, 1978), extensive re-solubilization of phenolic monomers occurred in the small intestine of our sheep. The potential for inhibition of intestinal bacteria by these phenolics must be considered based on in vitro values (Akin, 1982; Jung \& Fahey, 1983b). Whether the microflora of the ruminant digestive tract is susceptible to the low levels of soluble phenolics found there is unknown. If the $24 \mathrm{~h}$ rumen samples in Expt 2 are assumed to represent prefeeding levels of soluble phenolic monomers, the very high concentrations of these compounds at zero time, immediately after feeding, represent rapid solubilization of the compounds from the lucerne hay. This should be possible as forages contain phenolic monomers in the cell solubles (Jung et al. 1983). This interpretation is not valid for protocatechuic acid, however, as it is apparently found only in the cell wall fraction of forages (Jung et al. 1983). The secondary peak concentration at $12 \mathrm{~h}$ post-feeding for several of the compounds may represent release of phenolic monomers as a result of cell wall degradation.

The results reported here demonstrate that lignin undergoes extensive modification during its passage through the gastrointestinal tract of sheep. Phenolic monomers differ in their digestibilities among forages and sections of the gastrointestinal tract. This apparent digestion of lignin phenolics and the apparent absorption of phenolic monomers by ruminants indicate a potential for numerous interactions, both positive and negative, between these compounds and the biological processes of ruminant digestion and metabolism.

\section{REFERENCES}

Akin, D. E. (1980). Applied and Environmental Microbiology 40, 809-820.

Akin, D. E. (1982). Agronomy Journal 74, 424-428.

Allinson, D. W. \& Osbourn, D. F. (1970). Journal of Agricultural Science, Cambridge 74, 23-36.

Butler, J. H. A. \& Buckerfield, J. C. (1979). Soil Biology and Biochemistry 11, 507-513.

Chesson, A., Stewart, C. S. \& Wallace, R. J. (1982). Applied and Environmental Microbiology 44, $597-603$.

Crampton, E. W. \& Maynard, L. A. (1938). Journal of Nutrition 15, 383-395. 
Cymbaluk, N. F., Gordon, A. J. \& Neudoerffer, T. S. (1973). British Journal of Nutrition 29, 1-12.

Fahey, G. C. Jr, Al-Haydari, S. Y., Hinds, F. C. \& Short, D. E. (1980). Journal of Animal Science 50, $1165-1172$. Fahey, G. C. Jr, McLaren, G. A. \& Williams, J. E. (1979). Journal of Animal Science 48, 941-946.

Forbes, E. B., Elliot, R. F., Swift, R. W., James, W. H. \& Smith, V. F. (1946). Journal of Animal Science 5, 298-305. Gaillard, B. D. E. \& Richards, G. N. (1975). Carbohydrate Research 42, 135-145.

Gee, M. S., Nelson, O. E. \& Kuc, J. (1968). Archives of Biochemistry and Biophysics 123, 403-408.

Glick, Z. (1981). Journal of Nutrition 111, 1910-1916.

Goering, H. K. \& Van Soest, P. J. (1970). USDA Agricultural Handbook 379. Washington, DC:USDA.

Gray, D. H. \& Vogt, J. R. (1974). Journal of Agricultural and Food Chemistry 22, 144-146.

Hartley, R. D. (1971). Journal of Chromatography 54, 335-344.

Hartley, R. D. \& Buchan, H. (1979). Journal of Chromatography 180, 139-143.

Hartley, R. D. \& Jones, E. C. (1977). Phytochemistry 16, 1531-1534.

Hartley, R. D., Jones, E. C. \& Wood, T. M. (1976). Phytochemistry 15, 305-307.

Hartnell, G. F. \& Satter, L. D. (1979). Journal of Animal Science 48, 375-380.

Joslyn, M. A. \& Glick, Z. (1969). Journal of Nutrition 98, 119-126.

Jung, H. G. \& Fahey, G. C. Jr (1983a). Journal of Animal Science 57, 206-219.

Jung, H. G. \& Fahey, G. C. Jr (1983b). Journal of Dairy Science 66, 1265-1268.

Jung, H. G. \& Fahey, G. C. Jr (1983c). Journal of Nutrition 113, 546-556.

Jung, H. G., Fahey, G. C. Jr \& Garst, J. E. (1983). Journal of Animal Science (In the Press.)

Kane, E. A., Jacobson, W. C. \& Moore, L. A. (1950). Journal of Nutrition 41, 583-596.

Martin, A. K. (1982). British Journal of Nutrition 47, 155-164.

Muntifering, R. B., DeGregorio, R. M. \& Deetz, L. E. (1981). Nutrition Reports International 24, 543-549.

Neilson, M. J. \& Richards, G. N. (1978). Journal of the Science of Food and Agriculture 29, 513-519.

Rodwell, V. W. (1979). In Review of Physiological Chemistry, pp. $406-429$ [H. A. Harper, V. W. Rodwell and P. A. Mayes, editors]. Los Altos, California: Lange Medical Publications.

Scheline, R. R. (1978). Mammalian Metabolism of Plant Xenobiotics. London: Academic Press.

Theander, O., Uden, P. \& Aman, P. (1981). Agriculture and Environment 6, 127-133. 